\title{
Pierre BEAUCAGE
}

Anthropologue, professeur au département d’anthropologie, Université de Montréal

(1992)

\section{"Héros civilisateur ou oppresseur ridicule? La représentation de l'étranger dans la littérature orale maseual (nahuat) du Mexique”}

\author{
Un document produit en version numérique par Jean-Marie Tremblay, bénévole, \\ professeur de sociologie au Cégep de Chicoutimi \\ Courriel: jean-marie_tremblay@uqac.ca \\ Site web pédagogique : http://www.uqac.ca/jmt-sociologue/ \\ Dans le cadre de: "Les classiques des sciences sociales" \\ Une bibliothèque numérique fondée et dirigée par Jean-Marie Tremblay, \\ professeur de sociologie au Cégep de Chicoutimi \\ Site web: http://classiques.uqac.ca/ \\ Une collection développée en collaboration avec la Bibliothèque \\ Paul-Émile-Boulet de l'Université du Québec à Chicoutimi \\ Site web: http://bibliotheque.uqac.ca/
}




\section{Politique d'utilisation de la bibliothèque des Classiques}

Toute reproduction et rediffusion de nos fichiers est interdite, même avec la mention de leur provenance, sans l'autorisation formelle, écrite, du fondateur des Classiques des sciences sociales, Jean-Marie Tremblay, sociologue.

Les fichier des Classiques des sciences sociales ne peuvent sans autorisation formelle:

- être hébergé (en fichier ou page web, en totalité ou en partie) sur un serveur autre que celui des Classiques.

- servir de base de travail à un autre fichier modifié ensuite par tout autre moyen (couleur, police, mise en page, extraits, support, etc...),

Les fichiers (.html, .doc, .pdf., .rtf, .jpg, .gif) disponibles sur le site Les Classiques des sciences sociales sont la propriété des Classiques des sciences sociales, un organisme à but non lucratif composé exclusivement de bénévoles.

Ils sont disponibles pour une utilisation intellectuelle et personnelle et, en aucun cas, commerciale. Toute utilisation à des fins commerciales des fichiers sur ce site est strictement interdite et toute rediffusion est également strictement interdite.

L'accès à notre travail est libre et gratuit à tous les utilisateurs. C'est notre mission.

Jean-Marie Tremblay, sociologue

Fondateur et Président-directeur général, LES CLASSIQUES DES SCIENCES SOCIALES. 
Cette édition électronique a été réalisée par Jean-Marie Tremblay, bénévole, professeur de sociologie au Cégep de Chicoutimi à partir de :

Pierre Beaucage

[Anthropologue, professeur au département d'anthropologie, Université de Montréal.]

"Héros civilisateur ou oppresseur ridicule ? La représentation de l'étranger dans la littérature orale maseual (nahuat) du Mexique”.

Un article publié dans l'ouvrage sous la direction de Simon Harel, L'étranger dans tous ses états. Enjeux culturels et littéraires, pp. 81-98. Montréal : XYZ Éditeur, 1992, 190 pp. Collection : Théorie et littérature.

[Autorisation formelle accordée le 14 septembre 2007 par l'auteur de diffuser ce texte dans Les Classiques des sciences sociales.]

Courriels : pierre.beaucage@umontreal.ca

Polices de caractères utilisée :

Pour le texte: Times New Roman, 14 points.

Pour les citations : Times New Roman, 12 points.

Pour les notes de bas de page : Times New Roman, 12 points.

Édition électronique réalisée avec le traitement de textes Microsoft Word 2004 pour Macintosh.

Mise en page sur papier format : LETTRE (US letter), 8.5’’ x 11'’)

Édition numérique réalisée le 9 janvier 2008 à Chicoutimi, Ville de Saguenay, province de Québec, Canada.

\section{f Fait avec}




\section{Pierre BEAUCAGE}

Anthropologue, professeur au département d'anthropologie, Université de Montréal.

\section{"Héros civilisateur ou oppresseur ridicule? La représentation de l'étranger dans la littérature orale maseual (nahuat) du Mexique”}

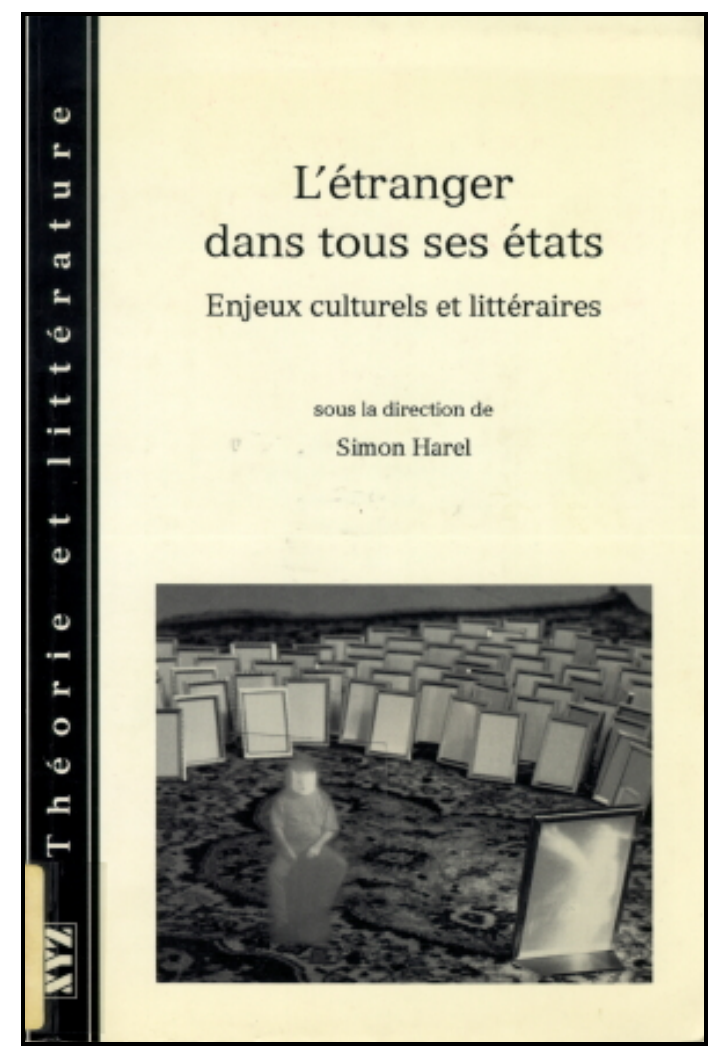

Un article publié dans l'ouvrage sous la direction de Simon Harel, L'étranger dans tous ses états. Enjeux culturels et littéraires, pp. 81-98. Montréal : XYZ Éditeur, 1992, 190 pp. Collection : Théorie et littérature. 


\section{Table des matières}

Introduction

La littérature orale maseual et ses genres

Le mythe d'origine (tajtol de in uejkaujkayot): l'émergence du Soi par essai et erreur

La rencontre d'êtres surnaturels (tanemililis)

Le récit ethnohistorique (tein techtapouiaj in tatajmej)

Le conte ordinaire (sanilmej, kuentos, tapouilis)

Les histoires de sexe (pitsotajtolmej)

L'étrange étranger 
Pierre BEAUCAGE

Anthropologue, professeur au département d'anthropologie,

Université de Montréal.

"Héros civilisateur ou oppresseur ridicule ? La représentation de l'étranger dans la littérature orale maseual (nahuat) du Mexique”.

Un article publié dans l'ouvrage sous la direction de Simon Harel, L'étranger dans tous ses états. Enjeux culturels et littéraires, pp. 81-98. Montréal : XYZ Éditeur, 1992, 190 pp. Collection : Théorie et littérature.

\section{Introduction}

$\underline{\text { Retour à la table des matières }}$

Parler du rapport à l'étranger, dans la Sierra Norte de Puebla ${ }^{1}$, au Mexique, c'est aborder la définition des rapports interethniques. De prime abord, ceux-ci présentent ce qu'on pourrait appeler une fausse simplicité : les autochtones nahuats ${ }^{2}$ de basse montagne se définis-

1 Les données qui servent de base à cet article ont été recueillies dans la Sierra Norte de Puebla au cours de divers stages de recherche, depuis 1969. Le texte a été établi après discussion entre P. Beaucage et les membres du Taller de Tradición Oral, association composée d'autochtones et de Métis qui se consacre à la collecte et à la diffusion de la tradition orale maseual et qui a son siège à San Miguel Tzinacapan, dans le municipe de Cuetzalan, Puebla. Fondé il y a dix ans, le Taller a à son actif une brochure sur les poèmes chantés du xochipitsaua, douze recueils de contes, un livre sur les plantes médicinales (en collaboration avec P. Beaucage) ; en attente de publication, un livre sur la tradition ethnohistorique, un autre sur le savoir zoologique et un troisième sur les plantes alimentaires. Les membres du Taller ont créé plusieurs pièces de théâtre qui illustrent les traditions et les conditions de vie actuelles des autochtones de la Sierra. Nous adoptons ici l'orthographe de la langue maseual établie par le Taller, en accord avec la linguiste Sybille Toumi et le ministère de l'Éducation du Mexique.

2 Les ethnologues appellent traditionnellement Nahuatl les Indiens qui parlent la langue qu'utilisaient les Aztèques et que parlent encore plus d'un million d'Indiens du Mexique. Les linguistes ont en outre créé le terme nahuat pour désigner la variante dialectale du sud-est de la Sierra de Puebla. Les princi- 
sent eux-mêmes comme maseualmej et renvoient tout ce qui n'est pas indien dans la catégorie koyot (fém. xinolaj, pl. koyomej). Ce terme comprend aussi bien les Métis du bourg voisin, établis depuis un siècle, que les gens des villes (catrines), les touristes (gringos), les rares natifs d'Espagne (gachupines), voire les envahisseurs français de 1860 (analtecos). Le mot koyot, clairement péjoratif puisqu'il se réfère à un animal considéré comme prédateur et parasitaire ${ }^{3}$, est généralement le premier qu'entend l'étranger quand il pénètre dans les villages indiens : crié par de tout jeunes enfants qui courent ensuite se réfugier dans les jupes de leur mère. Les dérivés du couple d'opposition maseual/koyot s'étendent à tous les secteurs de la vie culturelle et sociale : maseualtakualis, la nourriture indienne, s'opposant à koyotakualis, maseualtson, la musique indienne, à koyotson, etc. La structure de la langue maseual (maseualtaijol) permet d'ailleurs de créer constamment des néologismes, souvent à des fins humoristiques ${ }^{4}$.

paux intéressés ne se retrouvent pas dans ces noms, tombés en désuétude et qui pour eux rappellent fâcheusement naual («sorcier»). Le terme maseual par lequel ils s'autodésignent a la double connotation d'«Indien» et de « paysan » (cf. Pierre Beaucage, « Les identités indiennes : folklore ou facteur de transformation ", Construction / destruction sociale des idées : alternances, récurrences, nouveautés, B. Dumas et D. Winslow (sous la direction de), Actes du Congrès annuel des sociologues et anthropologues de langue française, Montréal, ACFAS, 1987).

3 Le fait de désigner justement l'étranger par un nom d'animal du pays pose problème. Un élément de compréhension peut venir du fait que dans le parler espagnol du Mexique, le terme coyote se rapporte à l'intermédiaire qui achète les récoltes des paysans pour les revendre à la ville. En outre, le lexicologue Santamaría nous dit que le terme désigna, sous la colonie, la personne née de l'union d'un Métis avec une Indienne, mais aussi que, lors de la guerre d'indépendance, on appela coyotes tous les Espagnols, dont plusieurs s'adonnaient au petit commerce de revente. (Francisco J. Santamaría, Diccionario de mejicanismos, México, Porrua, 1959, p. 225, 308).

4 Ainsi, l'ethnologue ayant fait remarquer que son bébé semblait aimer le pain (koyotaxkal. la tortilla-des-étrangers), le père répondit : « Il préférera bientôt la tortilla-des-Indiens » (masoualtaxkal), néologisme pléonastique qui souleva l'hilarité de l'assistance. Une autre fois, alors qu'il explicitait avec les membres du Taller le sens du mot xalkoyot (« un Indien qui essaie de passer pour Métis »), il se fit répondre : "C'est l'opposé de toi, qui serais plutôt xalmaseual!» 
L'opposition maseual/koyot n'éclaire cependant qu'une petite partie du champ sémantique de l'étranger dans la culture autochtone.

Le 31 octobre 1989, à la tombée de la nuit, j'arrive à San Miguel Tzinacapan, village indien où je retourne depuis 1984. À l'improviste, car la lettre annonçant mon arrivée s'est perdue en chemin. Par certaines portes encore ouvertes, on entrevoit les autels domestiques, richement décorés et couverts d'offrandes en cette veille de la Toussaint. Devant chaque maison, une traînée de pétales d'oeillets d'Inde (sempoualxochit, Tagetes erecta) indique aux âmes des défunts la route à suivre pour retrouver leur foyer et le banquet qu'on leur a préparé. Mais l'ethnologue frappe en vain à la porte de son compadre. Pourtant, il lui avait semblé entendre de la musique. De guerre lasse, il va se retirer quand finalement la porte s'ouvre et Pedro risque une tête dans l'embrasure. C'est l'éclat de rire. Mais un certain malaise demeure. On explique : «Ton double est venu avant toi !» Deux heures plus tôt, mon filleul avait mis par erreur sur le magnétophone une cassette avec la voix de l'étranger. Et voilà qu'il frappe à la porte! Les occupants du lieu en ont tiré la conclusion la plus plausible : mort au loin, l'ethnologue a entendu l'appel (tanot-salis) et vu la traînée de pétales. Mais en même temps il n'a rien à voir, en tant qu'étranger, avec la fête indienne des morts. Il se créait donc un désordre qui ne pouvait être que funeste.

Sans que l'ethnologue le veuille, son arrivée avait emmêlé deux niveaux d'identité/ altérité. jusqu'alors, il connaissait surtout le premier niveau, ethnique. Cependant, pour opposés qu'ils soient dans la vie sociale, maseual et koyot se rejoignent néanmoins dans la catégorie kristianoj, empruntée à l'espagnol : en nahuat, le terme perd sa signification originelle de " chrétien » pour désigner l'être humain, quelle que soit son appartenance d'ethnie, de genre ou de classe. À quoi s'oppose kristianoj ? Qui ou quoi n'est pas kristianoj ? La question provoque immanquablement la gêne chez l'interlocuteur autochtone. Ce n'est pas au règne végétal ou minéral qu'il pense, mais à une vaste 
zone que l'on désigne généralement par des circonlocutions à connotation fortement négative : collectivement, tein amo tokniuan (« ceux qui ne sont pas nos frères ») ou, au singulier : oksé («l'Autre »), in amo kuali (« le pas-bon »), in okuilin (« la bête ») 5 .

À ce second niveau, métaphysique, l'Autre, à la fois un et multiple, est perçu comme l'absolument différent, l'Étrange et l'Étranger par excellence, puisqu'il ne rejoint l'humain dans aucune catégorie supérieure. Pour le non-Indien, l'exploration de cet univers symbolique ne peut se faire que très progressivement, presque confidentiellement et de façon indirecte, à travers les récits où interviennent les êtres surnaturels. Car ce qui n'est pas humain est potentiellement très dangereux ; le nommer revient à l'appeler, ce qui peut déclencher des catastrophes. C'est pourquoi la transmission de ce savoir est aussi nécessaire que risquée, et doit s'accompagner de multiples précautions ${ }^{6}$. En effet, le monde surnaturel n'est pas situé dans un lointain au-delà, enfer ou paradis, mais est tangent au nôtre et le croise en plusieurs points de l'espace-temps : les carrefours, les grottes, la forêt de haute futaie, mais aussi les coupures de midi et de minuit, de la naissance et de la mort. L'étrange, dans toutes ses manifestations, signale cet Autre : des rochers en forme de piliers ou une eau sans cesse agitée indiquent un lieu maudit, le ululement d'un hibou qui passe et repasse appelle les âmes, un engoulevent qui vous «barre le chemin » est de mauvais augure.

Nous n'entreprendrons pas ici l'examen détaillé du surnaturel maseual. Signalons simplement qu'il comprend des êtres essentiellement

5 Maria Eugenia Sanchez, Temps, espace et changement social. Perspectives à partir de la communauté indigène de San Miguel Tzinacapan (Mexique), thèse de maîtrise, Paris, Centre de recherches coopératives-EHEES, 1978, p. 151, Tim Knab, Words, Great and Small : Sierra Nahuat Narrative Discourse in Everyday Life, thèse de doctorat, Albany, State University of New York, 1983, p. 80-81 ; Taller de Tradición Oral, Masoualsanilmej 2. Cuentas indígenas de la región de San Miguel Tzinacapan, Pue, San Miguel Tzinacapan, CEPEC, 1985, p. 20 et suiv.

6 Tim Knab, op. cit., p. ii-viii. 
maléfiques, comme les monstres (takauaksal, youalnenkej), les sorciers (naualmej) et les ogres (masakamej, tepeuanimej, auanimej), des êtres bénéfiques comme le Père et la Mère Nourriciers (teiskaltitoueytajtsin), le Maître du monde souterrain (talokanka) ainsi que des êtres ambigus comme les foudres (achiuanimej, kioujteyomej), les gnomes protecteurs des récoltes (talokej taxkaltianij) et les gardiens de l'eau (apiani) et des montagnes (tepeyolomej), etc. Chaque être humain possède une âme double (tonal) qui constitue son lien organique avec le surnaturel : le rêve témoignant de ses voyages ${ }^{7}$.

Dans les pages qui suivent, nous examinerons les diverses caractéristiques et les fonctions des représentations de l'étrange et de l'étranger telles qu'on les retrouve dans la littérature orale des Maseualmej de basse montagne.

\section{La littérature orale maseual et ses genres}

$\underline{\text { Retour à la table des matières }}$

Toute tentative de définition des genres dans la littérature orale maseual doit tenir compte de la hiérarchie qui existe quant à l'usage de la parole : ce n'est pas n'importe qui qui peut parler de n'importe quoi. Ni un jeune ni une femme ne se risqueront à raconter un récit important dont un aîné est dépositaire : tout au plus pourra-t-on en faire une version simplifiée pour amuser les enfants... ou les ethnologues. Seuls les fous « disent toutes sortes de choses, content toutes sortes de cho-

7 Voir Tim Knab, Geografía del inframundo, Montréal, Groupe de recherche sur l'Amérique latine (GRAL), 1990 ; María Eugenia Sánchez, op. cit. ; María Elena Aramoni, Bases cosmológicas del shamanismo entre los nahuas de la Sierra Norte de Puebla (Mexico), Montréal, Groupe de recherche sur l'Amérique latine (GRAL), 1988 ; Eliseo Zarnora-Islas, "L'âme captive et les quatre lieux de la terre : un récit nahuat de guérison », Culture, vol. VIII, no 2, 1988, p. 81-85. 
ses, mais ils ne savent pas, ils n'écoutent pas, ils n'ont pas la permission ${ }^{8} »$.

Les Maseualmej utilisent plusieurs termes pour désigner le discours : tajtol (de tajtoua, parler), le plus général, qui désigne à la fois la parole, le mot, la voix ; sanil, les "propos sans importance » mais aussi les contes, les récits ; tapouilis, « ce qu'on raconte »; tanemililis, " pensée, réflexion ». Cette multiplicité de termes recouvre des pratiques discursives différenciées. Knab a proposé de distinguer six catégories ou niveaux de discours :

- tajtol de in uejkaujkayot (" parole d'il y a longtemps »), le mythe d'origine, très vaste, généralement raconté de façon fragmentaire.

- tajtol melauj ("parole véridique »), histoire où des humains sont aux prises avec des esprits ou des revenants. Knab a utilisé le terme melauj par suite de l'affirmation constante des narrateurs que le récit relate des faits authentiques, et n'est pas un « conte ordinaire » (voir plus loin). Nous préférons le terme tanemililis (" pensée, réflexion ») qui semble d'usage plus courant ${ }^{9}$.

- tajtol tepitsin («parole petite ») : l'expression - laborieuse correspond au terme usuel sanil ("propos divers, conte ») et à ses équivalents : tapouilis, kuentoj, kuentitoj, « conte ». Nous utiliserons le terme sanil, attesté ailleurs dans Faire nahua ${ }^{10}$. Quantitativement les plus importants dans les corpus de récits mésoaméricains ${ }^{11}$, les contes mettent surtout en scène des humains et des animaux. Lors-

8 Tim Knab, Words, Great and Small, op. cit., p. 79-80.

9 Taller de Tradición Oral, Maseualsanilmej 8. Cuentos indigenas de la región de San Miguel Tzinacapan, Pue, San Miguel Tzinacapan, CEPEC, 1987, p. 6.

10 Cf. sasanilli dans Alfredo López-Austin, Los mitos del tlacuache, México, Alianza Editorial Mexicana, 1990, p. 282.

11 Robert M. Laughlin, Of Cabbages and Kings. Tales from Zinacantan, Washington Institute, 1977, p. 122. 
qu'un être surnaturel y intervient, il semble correspondre au Diable chrétien et son pouvoir y est tourné en dérision.

- tajtol tapalin ( « récit coloré ou peint »), histoire dont le thème central est la sexualité. L'expression employée par Knab semble une traduction littérale au maseual de l'espagnol cuento colorado (« conte rouge ou coloré »). Nous lui préférerons le terme pitsotajtol («parole cochonne »), un des termes les plus fréquents pour désigner les histoires de sexe.

Nous supprimons ses catégories ueytajtol ("parole longue », cycle de contes) et siuatajtol ("parole de femme », commérages) puisque ces derniers ne sont pas habituellement distingués des sanilmej (voir plus haut), mais nous ajoutons le récit ethno-historique, que les membres indiens du Taller de Tradición Oral de San Miguel ont euxmêmes désigné par la périphrase tein techtapouiaj in tatajmej («ce que les aïeux nous racontaient »).

Les genres de la littérature orale maseual. se distinguent essentiellement par le thème, le contexte et un certain nombre de marqueurs. Ainsi, les deux premiers (mythes et histoires de revenants) et le dernier (ethno-histoire) sont racontés par des aînés devant la famille, à laquelle ont pu s'ajouter des invités choisis ${ }^{12}$. À l'opposé, n'importe qui peut dire un conte. Ce dernier s'adresse surtout, mais pas exclusivement, à un public d'enfants, et les adultes présents y prêtent généralement grande attention. Si le talent de conteur est apprécié, par contre quelqu'un qui raconte trop, ou surtout, hors de propos, fera dire de lui tesaniluia ("C'est un hâbleur »). Quant aux histoires de sexe, elles se caractérisent par un langage cru, ne sont pas jugées convenables pour les femmes et les enfants et sont particulièrement à l'honneur là où

12 « Comment voulais-tu qu'on t'en parle à plusieurs alors que nous-mêmes n'en parlons pas en dehors de la famille ? » déclarera une Indienne à une ethnologue qui avait tenté d'amorcer une discussion de groupe sur le thème des forces surnaturelles (M. E. Sánchez, communication personnelle). 
plusieurs hommes travaillent ensemble (par exemple, pour la culture du maïs ou les corvées communautaires) ${ }^{13}$.

Notre hypothèse est que la littérature orale maseual n'offre pas une image de l'étranger, mais que cette dernière varie énormément d'un genre à l'autre, en rapport avec la fonction sociale spécifique de chacun. Une véritable démonstration exigerait une analyse exhaustive de centaines de mythes et de contes. Nous nous limiterons à illustrer notre thèse au moyen de quelques récits que nous considérons représentatifs des divers genres.

\section{Le mythe d'origine (tajtol de in uejkaujkayot) : l'émergence du Soi par essai et erreur}

$\underline{\text { Retour à la table des matières }}$

Pour les Nahuats actuels comme pour leurs ancêtres précolombiens ${ }^{14}$, le monde actuel ne fut pas créé tel quel, par suite de l'action de quelque démiurge. Plusieurs ères-mondes se sont succédé, au cours desquels apparurent, disparurent et se transformèrent les éléments, les plantes et les animaux, les humains et les êtres surnaturels. Aujourd'hui encore, un vaste ensemble de récits, lâchement intégrés, rendent compte de cette cosmogénèse. Nous avons choisi le mythe de Sentiopil (« le fils du maïs ») pour illustrer les rapports du soi et de l'autre que pose ce type de discours :

13 Il y a cependant des limites à ne pas dépasser. Un groupe d'hommes, réunis pour le sarclage d'une milpa, rivalisaient de pitsotajtolmej quand l'un d'eux s'écria soudain : kouat! (le serpent). L'apparition du messager des esprits fut interprétée comme un rappel à l'ordre et le reste du travail s'effectua en silence (E. Salazar, communication personnelle).

14 Voir la «légende des quatre soleils» (Códice Chimalpopoca) qui relate les créations et fins du monde successives, ainsi que l'analyse récente d'Alfredo Lopez-Austin, Los mitas del tlacuache, México, Alianza Editorial Mexicana, 1990. 


\section{Sentiopil (extraits)}

Il y a longtemps (uejkauj) vinrent à vivre certains de nos frères (tokniuan ${ }^{15}$ ) qui s'appelaient ogres (tsitsimimej). Ils se mangeaient les uns les autres, et c'est pourquoi on les appelait ainsi. Un colibri connut (= tomba amoureux d') une jeune ogresse. Il ne pouvait pas causer avec elle (= arranger l'union) mais il la piqua et tira un peu de son sang. Il le mêla avec le sien et le jeta au bord de la fontaine. Une plante domestique (tatok) y poussa, donna son fruit et personne ne le connaissait. Les anciens l'ouvrirent et virent qu'il avait du sang. Ils le jetèrent dans la fontaine. Ensuite ils vinrent et virent un bébé qui pleurait : ils le tirèrent de là et l'emmenèrent chez eux. Là il grandit et on l'appela « fils du mais ». Plus tard il apprit la musique et jouait pour les rendre contents. Et les ogres disaient : "Réjouis-nous, on te mangera quand même. » [Ils essayèrent de le brûler dans le bain de vapeur, mais il se protégea avec deux tortues et c'est lui qui les brûla]. Seuls demeurèrent sa mère, des chiens et des enfants. Il ne connut pas son père et pensa à le reconstituer. Il demanda aux enfants : "Où avez-vous laissé les os de mon père ? » Ils répondirent : « Nous ne le savons pas. » Alors, il demanda à tous les chiens de retrouver les os. Il façonna son père et vint dire à sa mère : " J'ai façonné mon père, je l'apporte, mais ne regarde pas. » La mère n'a pas résisté et a regardé. Et dans les mains de Sentiopil s'est détruit son père. [Il réprimanda sa mère, puis décida de semer du maïs. Tous les animaux l'aidèrent.] Ils portèrent la récolte dans une grotte qui s'appelle : «Sur-lamontagne-grenier » (kueskomatepek). Puis il pensa à construire deux villages. Il fit México et Puebla. Il ne tarda pas à savoir que la lumière allait venir. Il dit : « le n'attendrai pas qu'il fasse jour, j'irai au fond de la mer. » [Plus tard, voyant que les deux villes sont habitées, il alla pour emprunter de l'argent aux habitants, qui, orgueilleux, lui refusèrent et le menacèrent. Il se fâcha et fit jaillir une rivière qui inonda tout].

Par rapport au thème qui nous occupe, le mythe montre une inversion des rapports du soi et de l'autre, qu'on retrouve dans d'autres récits du même genre ${ }^{16}$. Les premiers habitants sont à la fois des hu-

15 D'après la version en nabuat standard publiée par le Taller de Tradición Oral (1984). Le conteur avait quant à lui utilisé le mot espagnol anteriores ( «ancêtres »), qui possède le même sens d « humanité ».

16 Comparer avec le mythe parallèle de Tamakasti (Jesús Monjarás-Ruiz, Mitas cosmogónicos del México indígena, Mexico, Instituto Nacional de Antropología e Historia, 1987, p. 194-198). 
mains (ils aiment la musique, les bains de vapeur, sont pris de curiosité) et n'en sont pas : ils ignorent la nourriture végétale (le maïs) et consomment la chair humaine. La référence à la nourriture des personnages est fondamentale, car c'est le dis-moi-ce-que-tu-manges qui permet généralement de distinguer «nos frères » des autres, dans les mythes et les contes. Le héros, quant à lui, est l'élément étrange/ étranger, dans ce monde anormal : il invente la culture, sous la double forme de la musique et de l'agriculture. Mais en même temps, il est inassimilable à l'humanité : il converse et travaille avec des animaux, il est né du corps d'un homme, le plant de maïs, et sa grand-mère est en même temps... sa mère ! En outre, il ne cherche pas de compagne ; c'est pourquoi des enfants d'ogres doivent survivre, et devenir sans doute les habitants des deux villages qu'il a construits. C'est à ces derniers, imparfaits et dépendants, que les humains d'aujourd'hui peuvent s'identifier, et non à Sentiopil le surhomme. Il faut noter que comme les autres cycles de création, celui de Sentiopil se termine par une « fin du monde » : mais le maïs, créé, a été enfermé et c'est une nouvelle humanité qui le découvrira.

L'examen du mythe de Sentiopil nous permet de proposer que le discours maseual sur l'origine est caractérisé par une inversion répétée des pôles d'identité/ altérité.

\section{La rencontre d'êtres surnaturels (tanemililis)}

$\underline{\text { Retour à la table des matières }}$

Nous résumerons trois récits, qui permettront de dégager un modèle : l'ivrogne, le paysan paresseux, et le chasseur bigame. 


\section{L'Ivrogne et les masakamej ${ }^{17}$ (résumé)}

À la nuit tombée, un homme rentre ivre du bourg. Il approche de sa maison lorsque surgissent trois masakamej : "Viens par ici, c'est le bon chemin. » Ils le mènent en fait à une caverne et le poussent dedans. Là, dans l'obscurité, dans le lieu des morts, on le fait travailler, transporter des os, de la boue, des chairs pourries pour les masakamej. Il se souvient de son paquet de tabac et fume. Les esprits s'écartent et grâce aux chauves-souris, il retrouve la sortie. Rentré chez lui, sa femme est incrédule : « Tu mens ! Personne n'échappe aux masakamej. Avec qui as-tu passé la nuit ? » Mais bientôt sa fille tombe gravement malade : aucun remède ne peut la sauver. Puis il meurt à son tour. Les masakamej l'avaient suivi sous forme du Vent Mauvais (amokuali ejekat) qui pénètre les corps et tue 18 .

\section{Le Chasseur et le tepeuani (résumé)}

Un homme vit de la chasse. Avec son chien, il attrape les bêtes de la forêt, les dépèce, les fait boucaner et puis retourne voir nos frères et leur vend la viande. C'est un dur travail, dans des endroits dangereux pour les gens. Alors qu'il faudrait un homme et [= pour] une femme, lui, il a deux femmes : il vit avec l'une, puis avec l'autre. Un jour, son chien disparaît. Il suit sa trace jusqu'à une grotte. Là un tepeuani lui dit : « Mes animaux dans les enclos sont malades. Amène-moi ta femme et je te rends ton chien. » Le chasseur accepte. Il dit à sa femme : «Viens m'aider à boucaner la viande, dans la forêt. » Il l'emmène à la grotte. Le tepeuani l'a donnée en nourri-

17 Les masakamej («hommes-cerfs ») et les tepeuanimej («maîtres des montagnes ») sont des êtres à forme humaine qui habitent les cavernes du monde souterrain. Us masakamej en sortent, la nuit, pour détourner les voyageurs du chemin et les entraîner dans les grottes où ils les dévorent (Tim Knab, Words, Great and Small, op. cit., et Geograía del inframundo, op. cit.). Les tepeuanimej demeurent sous la terre, où ils s'occupent des doubles animaux (tonalmej) conservés dans « les quatorze enclos ». Ils s'irritent tout particulièrement lorsqu'on veut tailler des routes dans les montagnes (ibid.), où lorsque le comportement répréhensible d'un humain rend les animaux malades. Les deux types de créatures sont liée aux cycles de création/ destruction du monde. En effet, de nombreux mythes mésoaméricains mentionnent qu'un cerf a été le père des premiers hommes. Et un mythe maseual relate comment les tepeuanimej sont des (proto)humains qui n'ont pas voulu accepter la lumière du soleil et se sont réfugiés sous terre (Taller de Tradición Oral, Massualsanilmej 2. Cuentos indígenas de la región de San Miguel Tzinacapan, Pue, op. cit., p. 18-19).

18 Tim. Knab, Words, Great and Small, op. cit., p. 336-339. 
ture aux bêtes de la forêt que le chasseur avait chassées. Et lui, en échange, il a eu des animaux. Et il a bien vécu, il n'avait plus deux femmes 19 .

\section{Le, Paresseux, les Tonnerres et les Vautours (résumé)}

Un homme restait là toute la journée, et ne sortait que pour jouer, se promener, se divertir, n'achetait rien à sa femme et ne lui donnait pas non plus un sou pour qu'elle puisse subsister. Elle devait moudre du grain chez les autres, travailler à la journée ; on lui offrait des tortillas et de la soupe et elle en gardait pour donner à son mari. Elle l'envoie aux champs mais il se couche à l'ombre. Voyant des éclairs, il se dit qu'il aimerait bien être un tonnerre (kioujteyot) pour lancer des éclairs dans le ciel. Son voeu est exaucé, mais une fois revêtu du brillant costume, il ne sait que provoquer des catastrophes. De retour sur terre, mais pas guéri de sa paresse, il aperçoit des vautours et il se dit qu'il aimerait voler ainsi et pouvoir manger sans travailler. Un vautour change de " costume » avec lui mais là il découvre qu'il lui faudra se nourrir de charogne et d'ordures. Sa femme s'accommode assez bien de son successeur qui se révèle être un mari malodorant, niais travailleur et généreux ${ }^{20}$.

Le sens des tanemililis apparaîtra assez clair. La fonction des êtres surnaturels est de sanctionner les écarts graves dans le comportement des humains : pour l'homme, ce sera s'enivrer au bourg plutôt que rester avec les siens, courir les femmes dans une société où le mariage monogamique s'inscrit dans l'ordre cosmique, et refuser de pourvoir à l'alimentation de sa famille. D'ailleurs, les narrateurs explicitent souvent, au début ou à la fin du récit, sa moralité. Doña Rufina, chamane et conteuse, commençait ainsi le récit du paresseux :

Écoutez-moi, grands et petits, maintenant je vais vous dire une histoire (tanemililis : une réflexion), que ce n'est pas bon d'être paresseux car la paresse est mauvaise. Celui qui ne fait rien se laissera tenter: tu seras ivrogne, tu prendras le goût de boire, et tu te bagarreras ou tu voleras. Et pour que ça n'arrive pas, écoutez bien l'histoire du paresseux ${ }^{21}$...

19 Ibid., p. 336-352.

20 Taller de Tradición Oral, Maseualsanilmej 8. Cuentas indigenas de la región de San Miguel Tzinacapan, Pue, op. cit., p. 7-43.

21 Ibid., p. 6-7. 
Dans ces contes moraux, on voit que l'étrange n'interfère avec le quotidien que dans la mesure où ce dernier est déjà perturbé par la transgression des normes. Il faut noter que cet étrange n'est pas vraiment étranger. Ce sont les esprits de la terre même qui interviennent ; et le Talokan, le monde souterrain où résident tous les esprits, est indien. Les forces qui l'habitent comprennent la langue indienne à l'exclusion de toute autre et ne frappent les étrangers que lorsque ceux-ci s'attaquent aux fondements mêmes de la vie sociale et culturelle : en abusant des Indiens ou en entaillant le flanc des montagnes pour construire des routes. Par ailleurs, les autochtones ne manifestent guère de préoccupation pour d'éventuelles punitions dans l'au-delà des chrétiens. De même, face à l'épouvante (nemoujtil) causée par les esprits, la médecine occidentale s'avérera impuissante : seuls les rituels autochtones pourront apaiser les dieux et guérir les malades ${ }^{22}$. On note aussi que le collectif prime sur un concept de responsabilité morale strictement individuelle : l'ivrognerie du premier personnage a entraîné aussi la mort de sa fille, tandis que le chasseur a résolu son problème de bigamie en sacrifiant une épouse aux génies chtoniens !

\section{Le récit ethnohistorique (tein techtapouiaj in tatajmej)}

$\underline{\text { Retour à la table des matières }}$

Le récit ethnohistorique prétend également à la vérité. Non plus sanctionnée par les êtres surnaturels, cependant, mais par l'âge et l'expérience du narrateur. Ce dernier émaillera son récit de références topographiques et chronologiques, de la même manière que les conteurs

22 Voir Maria Eugenia Sánchez, Temps, espace et changement social, op. cit., p. 153 et suiv. ; Diane Bilodeau, "Le susto : faiblesse ou maladie de l'âme ? " Recherches amérindiennes au Québec, vol. XI, no 1, 1981, p. 34-40 ; Enzo Segre, Las máscaras de Io sagrado, México, Instituto Nacional de Antropología e Historia, 1987, Eliseo Zamora-Islas, loc. cit. 
précédents entrecoupaient les récits de détails sur les esprits et les monstres à l'œuvre. (Les deux fragments qui suivent proviennent du corpus recueilli par le Taller de Tradición Oral et sont en voie de publication.)

\section{L'Arrivée des Métis}

Quand les Métis sont arrivés [à Cuetzalan, le bourg actuel], ils nous disaient : " Loue-moi ton terrain et va travailler sur ta ferme, j'en prends soin. Sois sans crainte, tu reviens, comme toujours, et c'est ta maison. » Nos gens leur ont fait confiance, pas vrai, ils leur ont donné la permission et beaucoup de gens les ont acceptés. Les années ont passé, peut-être dix ans. Certains, qui étaient de bonne foi, ont respecté les ententes, mais d'autres pas. Ils leur ont dit : « La maison ici est à moi, parce que la loi dit que quand je reste longtemps, c'est à moi. Tu dois le reconnaître. » [...] Et certains ont dit: «D'accord, je vais te l'acheter, ton lopin. » Mais ils l'achetaient si bon marché que ça n'était pas valide, pas vrai ? Et c'est ainsi que presque tout le monde a dû partir de là 23 .

Mais si les Indiens se rappellent la fin du XIXe siècle comme une période d'oppression, la Révolution de 1910-1917 (kuejmol) signifia d'abord pour eux des vexations sans nombre, et ce, des deux côtés :

\section{La Révolution, et après}

Les villistas [partisans de Pancho Villa] volaient nos mulets, nos bouvillons, tout ce qu'ils trouvaient : ta couverture, tes vêtements, ton argent, ils emportaient tout [...] Mon père nous racontait que quand ils croisaient des jeunes filles, ils abusaient d'elles, ils violaient toutes les femmes. C'est ce que faisaient les villistas [...] Parfois, en janvier, quand les femmes vont porter le repas aux hommes dans les champs, les soldats [progouvernementaux] les cherchaient pour abuser d'elles et ils volaient les tortillas qui devaient être pour les paysans [...] Ce qu'ils pouvaient nous détester, bon Dieu ! [...] Nos frères couraient comme des fourmis noires se cacher dans les cavernes, pour dormir là-bas, sans eau, sans sucre de canne, sans rien. Personne ne faisait de feu, on ne pouvait pas cuire les tortillas. Ça a été notre vie! On en a enduré, bon Dieu ! Et les autorités ne

23 Taller de Tradición. Oral, «Et ensuite, on n'a plus eu peur, témoignages nahuas de la région de Cuetzalan (Puebla, Mexique)», Anthropologie et Sociétés, vol. XI, no 2, p. 118. 
nous aidaient pas. Au lieu de nous aider, ils envoyaient des délégués pour ramasser des tortillas, de force, pour les soldats [...].

Si c'était aujourd'hui, on les aurait battus bien avant parce qu'aujourd'hui les jeunes n'ont plus peur. Avant, beaucoup avaient peur. le parle de ce temps d'où nous venons : les gens étaient respectueux et peureux. Aujourd'hui, il n'y a plus de peureux [...] Même un adolescent pourra te lancer une pierre et te tuer 24 !

Avec le récit ethnohistorique, l'Autre se définit désormais essentiellement en termes ethniques : c'est le conquérant espagnol, l'envahisseur français, le commerçant, le « révolutionnaire » et le soldat métis. À ce niveau, la relation maseual/koyot polarise tout le discours, effaçant presque complètement les différences internes à l'intérieur de chacune des deux catégories. Mais les rapports entre ces deux groupes, tels qu'ils se dégagent des récits de ce genre, sont ambigus. D'un côté on y relate dans le détail, en les dénonçant, les exactions multiples subies par le groupe aux mains de l'ethnie dominante : d'où une sensation d'impuissance. Par ailleurs, et en même temps, les Maseuals de la Sierra sont conscients d'avoir modifié avec le temps leur rapport avec les non-Indiens : autrefois, nous obéissions, maintenant, on a plus peur. La possibilité de la rébellion, du renversement du rapport avec l'autre, y est également inscrite. (L'actuel mouvement paysan autochtone, la coopérative régionale Tosepan Titataniske, apparaît ainsi directement enraciné dans les précédents historiques de contestation.)

\section{Le conte ordinaire (sanilmej, kuentos, tapouilis)}

$\underline{\text { Retour à la table des matières }}$

La littérature orale maseuale présente une variété considérable de contes ordinaires. Beaucoup mettent en scène des animaux et constituent des cycles centrés sur un trickster ou joueur de tours : le lièvre et

24 lbid., p. 118-120. 
l'opossum en sont les héros favoris et réussissent à se moquer des chasseurs, des chiens et des gros carnassiers comme le coyote et le jaguar. Ceux qui nous intéressent ici mettent surtout en scène des êtres humains : la condition indienne, ses contradictions et sa reproduction.

\section{Les Trois Frères (résumé)}

C'étaient trois frères qui vivaient à la ferme, comme nous vivons tous sur nos fermes. Ils enviaient ceux qui gagnaient de l'argent rapidement et pas eux : eux se fatiguent et sont pauvres, ils gagnent péniblement quelques sous, se déchirent aux ronces et se font piquer par les fourmis. Les Métis, quant à eux, parlent espagnol, savent beaucoup de choses, savent lire, travaillent peu et gagnent beaucoup d'argent. Mais avant tout, pour accéder à ce « travail de Métis » (koyotekit), ils décident d'apprendre l'espagnol : au bourg métis, chacun écoute attentivement et retient une phrase, sans en savoir le sens. Puis ils liquident leurs biens et vont s'installer en tant que licenciados (avocats). Un meurtre est commis, ils accourent, désireux de se faire une clientèle. Aux questions des policiers, ils répondent à contresens et se font coffrer pour de bon ${ }^{25}$.

\section{Les Trois Orphelins (résumé)}

Une dame eut trois fils et ils grandirent. Quand leur mère mourut, ils s'attristèrent, car il n'y avait plus personne pour leur faire les tortillas, leur laver le linge : ils étaient orphelins. L'aîné leur dit : « Séparons-nous. Partageons-nous les quelques sous qui restent pour acheter les outils dont nous aurons besoin pour travailler. Dans sept ans nous reviendrons mettre ensemble nos économies pour nous faire une maison. » Le cadet décide d'être avocat et s'achète un crayon, le second veut être voleur et s'achète une arme, l'aîné se procure une machette pour être journalier. Sept ans plus tard ils se retrouvent, sous le même arbre où ils ont tracé une croix. L'avocat est un crève-la-faim, le voleur n'a jamais été tranquille, seul le journalier a réuni quelques économies. La mort frappe les deux premiers, qui n'ont pas pu ramasser de quoi s'établir, ce à quoi ils s'étaient engagés 26 .

25 Taller de Tradición Oral, Maseualsanilmej 5. Cuentas indigenas de la región de San Miguel Tzinacapan, Pue, op. cit., p. 6-21.

26 Taller de Tradición, Oral, Maseualsanilmej 9. Cuentos indígenas de la región de San Miguel Tzinacapan, Pue, op. cit., p. 28-43. 


\section{Les Mariés et la Rose}

Un jeune couple se jure l'amour éternel. À tel point que, la femme étant décédée, l'homme demande à être enterré avec elle. Une souris lui apporte alors une rose qui permet de la ressusciter. Mais la jeune mariée, volage, le quitte pour épouser un roi. Le mari erre sur les routes. Un jour, il apprend que la fille d'un autre roi est morte : il va au palais et la ressuscite. « Putain d'Indien ", s'écrie-t-elle à son réveil en le voyant près d'elle. Mais son père lui apprend qu'il lui a sauvé la vie et qu'elle doit l'épouser. Devenu roi, il déclare la guerre à celui qui lui avait pris son épouse ; vainqueur, il fait capturer, exécuter et brûler sur un bûcher et son rival et l'épouse infidèle 27 .

\section{Le Paresseux et les Voleurs}

Un garçon vivait à ne rien faire, il ne voulait pas sortir, pas même pour aller chercher du bois. Il passait ses journées assis, à se chauffer les pieds sur les pierres du foyer. Son oncle commerçant allait au marché à tous les huit jours et en ramenait de l'argent. "Comment fais-tu ? », demanda le paresseux. L'oncle répondit: «je ramasse la cendre et on me l'achète en ville. » Le neveu remplit un sac de cendres et se rend au marché, où il attend en vain les clients. «Pauvre garçon, dit un passant, les gens achètent le tequisquite ${ }^{28}$, pas la cendre. je t'en donne trente centavos, pour que tu ne sois pas venu pour rien. ", Avec l'argent, le garçon s'achète un masque de démon. Le soir, il bivouaque sur la route. Mais les muletiers qui l'entourent sont en fait des voleurs. À minuit, l'envie lui prend d'essayer son masque. Quand les voleurs le voient, ils croient l'heure du châtiment venue et déguerpissent, lui laissant leurs bêtes et leur butin. Il revient au village et vit désormais à l'aise 29 .

Les résumés qui précèdent illustrent assez bien les deux tendances présentes dans le conte maseual, comme dans beaucoup de contes populaires. Les Trois Frères et Les Trois orphelins défendent l'ordre so-

27 Taller de Tradición Oral, Maseualsanilmej 8. Cuentas indigenas de la región de San Miguel Tzinacapan, Pue, op. cit., p. 6-39.

28 Mélange naturel de carbonate de soude et de chlorure de sodium, utilisé dans la cuisine et la pharmacopée populaires (Francisco J. Santamaría, op. cit., p. 1036).

29 Taller de Tradición Oral, Maseualsanilmej 11. Cuentos indigenas de la región de San Miguel Tzinacapan, Pue, San Miguel Tzinacapan, 1989, p. 6-27. 
cial existant, tout comme les récits moralisateurs où intervenaient les êtres surnaturels. Le premier soutient implicitement qu'il faut maintenir la différence ethnique et que les Indiens ne doivent pas essayer de copier les étrangers ; le second, qu'il faut être honnête et travailleur : tant pis pour les avocats et les voleurs! Seuls leurs styles diffèrent : le premier procède par la voie du sarcasme 30 tandis que le second adopte un ton dramatique. Dans Les Mariés et la Rose, on retrouve la même intention moralisatrice (punition exemplaire de la femme adultère) mais qui se combine cette fois avec un élément tout à fait subversif : le jeune Indien épouse la fille du roi. Il est intéressant de noter que le mariage se fera, visiblement, contre le gré de la princesse. Une idylle aurait pu sembler par trop invraisemblable, ou alors vouée à une fin tragique, vu l'importance des préjugés ethniques. Mais une princesse obligée d'épouser un pauvre Indien à cause de sa magie, voilà pour venger du mépris des jeunes métisses du bourg !

Dans Le Paresseux et les Voleurs, nous voyons comment le contenu du conte peut tendre à subvertir complètement les normes fondamentales de comportement établies dans les tanemililis. Loin d'être la mère de tous les vices, la paresse peut être la clef du succès, à condition d'avoir de bons réflexes... et un peu de chance. Les contes de ce genre reprennent sur le mode mineur du rire ce que le récit ethnohistorique affirmait sur un mode majeur, à savoir que la vie sociale est un mélange de déterminations subies et d'espaces ouverts à la contestation et à l'initiative. On retrouve là l'un des leitmotivs des histoires d'animaux, dans lesquelles un trickster, tour à tour victime et rebelle, bastonnant et bastonné, joue un rôle central ${ }^{31}$.

30 Les membres du Taller en ont d'ailleurs tiré une pièce de théâtre qui remporte toujours un vif succès dans les communautés.

31 Voir Pierre Beaucage, "Les animaux dans les mythes », L'oeil amérindien, Hélène Dionne (sous la direction de) Québec, Musée de la civilisation, 1991, p. 31-52. 


\section{Les histoires de sexe (pitsotajtolmej)}

$\underline{\text { Retour à la table des matières }}$

Elles sont, on peut l'imaginer, innombrables. Mais l'étranger ethnologue, fait face à deux difficultés : a) s'en faire raconter une et b) si on vous l'a racontée, bien la comprendre. En effet, le genre est considéré comme strictement interne à l'ethnie et la symbolique employée est souvent hermétique. Ainsi, il n'est pas immédiatement clair pour un occidental que l'avocatier ou le dindon renvoient au membre viril ! L'étranger n'a généralement droit qu'à des obscénités stéréotypées du genre iXinechtaneuj mouelti! (Prête-moi ta petite sœur !) qui semblent avoir surtout pour but de vérifier sa compétence linguistique. Si en plus il est ethnologue, une deuxième censure s'impose : et s'il allait raconter tout ça dans un livre, quelle idée auront de nous les gens de son pays ? La plupart des histoires sont brèves comme La Marchande et l'Étranger, mais certaines sont plus élaborées, comme Le Diable et le Gardien de bœufs, et Maria.

\section{La Marchande et l'Étranger}

C'est une pauvre femme, veuve, avec deux enfants en bas âge. Elle vit dans une chaumière, elle est pauvre mais courageuse. Elle ramasse des herbes sauvages et elle achète des fruits au village, pour les revendre au bourg. C'est ainsi qu'elle nourrit ses enfants. Un jour elle a apporté des bananes au marché, à Cuetzalan, avec ses deux enfants. L'un d'eux défèque. Elle ne peut laisser son éventaire : elle enveloppe rapidement l'ordure dans une pelure. Arrive un étranger : "Viens avec moi, tu n'as jamais vu ce que j'ai à t'offrir. » (Suit un dialogue salé inspiré par le produit en vente.) « D'accord, dit enfin la femme à l'étranger de plus en plus pressé, mais tu dois d'abord m'acheter quelque chose, un fruit au moins. » On devine quel fruit il paie rubis sur l'ongle et engloutit en moins de deux 32 .

32 Tim Knab, Words, Great and Small, op. cit., p. 281-285. 


\section{Le Diable et le Gardien de boeufs}

C'était un homme qui gardait les boeufs. Un jour, dans le pâturage, il vit un taureau maigre et décida de le châtrer pour qu'il engraisse. Arriva un monsieur à cheval, bien maigre, et qui lui dit :

- Que fais-tu là ?

- Je châtre mon taureau pour qu'il engraisse.

- Penses-tu que je pourrais engraisser si tu me châtrais ?

- Bien sûr que je peux !

- Alors c'est bon, châtre-moi. Et si dans trois mois je n'ai pas engraissé, je te châtre aussi et je t'emmène.

Ils firent l'accord, l'homme les lui a coupées et il est parti. Mais l'homme devenait bien triste parce qu'il savait que le diable, parce qu'il est mauvais, ne peut pas engraisser, et il voyait se rapprocher l'échéance.

Qu'est-ce que tu as ? lui demanda sa femme.

Il y a que ce monsieur, qui est le diable, va venir me châtrer aussi.

- Alors laisse-moi faire.

Quand le jour fut arrivé, la femme coupa ses cheveux, et mit le pantalon, la chemise et les bottes de son mari. Et elle partit rencontrer le diable.

- Je suis venu te châtrer, lui dit le diable, car je suis toujours aussi maigre.

- Il y a plusieurs jours que je le sais, c'est pourquoi je me suis castrée moimême.

Et elle lui montra au diable, pour qu'il voie. Et c'est comme ça qu'elle a sauvé la vie de son mari. Quand au diable, depuis, il va châtré 33.

33 Taller de Tradición Oral, inédit. 


\section{Maria (résumé)}

Il y avait un riche patron qui habitait une grande maison. Si grande qu'il y avait une pièce seulement pour se baigner. Ce patron avait trois jolies filles. Nul ne les approchait et il avait même châtré ses pistoleros pour qu'il ne leur arrive rien. Un jeune homme n'avait pas de terres, pas de travail. Il demande pour travailler à l'hacienda. On lui dit que le patron n'embauche pas de journaliers mais qu'il a besoin d'une cuisinière. Le jeune homme se déguise en femme et travaille un an à la cuisine, à moudre le mais. Il a pris l'habitude de moudre et a de bons bras. Il s'est fait appeler Maria ${ }^{34}$. Ensuite, on le prend au service de la maison. Quand les filles du patron se baignent, il leur apporte leurs vêtements et les séduit l'une après l'autre. Le patron voit que ses filles sont grosses et il est furieux. Il appelle à son tour « Maria » dans la salle de bains, bien décidé à en abuser. " Maria » profite de l'obscurité, met une chèvre à sa place, saisit le pistolet du patron et le tue d'un coup de feu à l'anus 35 .

L'univers des pitsotajtolmej, en autant qu'on puisse en juger par ce qu'on en sait, est celui de la subversion pure : l'improbable devient la norme, ce sont toujours les pauvres, les femmes et les Indiens qui l'emportent et ils (elles) infligent aux riches, aux machos et aux étrangers (incluant le diable chrétien) d'humiliantes - et généralement douloureuses - défaites. En ce sens, l'histoire de sexe est l'envers du mythe d'origine et du conte moral, qui définissent les fondements idéologiques de la vie sociale : elle récuse les déterminismes du pouvoir et pose la possibilité d'un renversement de l'ordre, possibilité déjà perceptible dans le récit ethnohistorique et le conte.

34 Nom que donnent les Métis à toutes les femmes indiennes. Il faut noter que jusque vers 1975, dans la Sierra, le nom d'une femme n'appartenait pas au domaine public et son usage était réservé à un groupe très intime. Si un enfant apprenait dès son jeune âge le nom de son père, il ne connaissait celui de sa mère qu'à l'adolescence.

35 Tim Knab, Words, Great and Small, op. cit., p. 285-290. 


\section{L'étrange étranger}

Retour à la table des matières

À travers les cinq genres de la littérature orale nahuat, le rapport à l'étrange/ étranger se modifie considérablement. Les mythes d'origine définissent des mondes où l'étrange constitue le cadre du quotidien : ce sont des éléments étrangers à ce cadre (animaux mythiques, démiurges) qui feront progresser l'univers en direction de la réalité actuelle. Cette réalité est bien établie dans le tanemililis, mais les normes sont souvent enfreintes par les humains : l'étrange et l'étranger fusionnés, sous la forme d'êtres surnaturels, interviennent pour sanctionner les écarts. Dans deux autres genres, ethnohistoire et conte, l'étranger devient l'ethnie dominante : les récits indiens illustrent et dénoncent la domination, la contestation occupe une place secondaire. C'est le rire maseual, on l'a vu avec les histoires de sexe, qui remet en question l'ensemble de la structure. L'étranger présomptueux et dominateur est débouté comme le patron, le curé et le mari : en même temps qu'une prise de distance s'opère par rapport au normal, à l'usuel, l'étrange et l'interdit deviennent familiers. Les lectures de la réalité que proposent les différents genres de la littérature orale indigène sont donc dans une tension constante, dans un équilibre de contradictions les unes par rapport aux autres : rien de plus éloigné de la simplicité répétitive que la pensée occidentale attribue encore si souvent à la " pensée sauvage ».

\section{Fin du texte}

